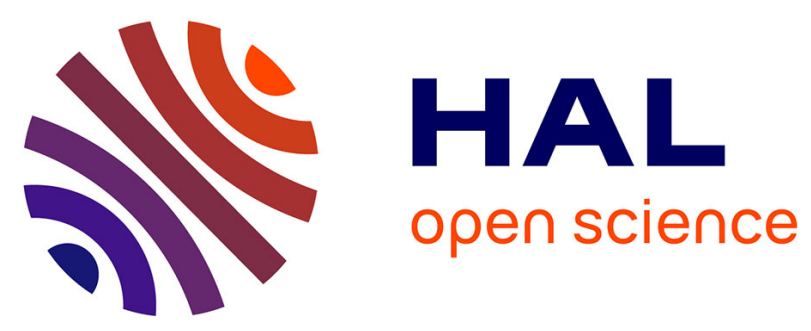

\title{
Design of an active fault tolerant control for nonlinear systems described by a multi-model representation
}

Mickael Rodrigues, Didier Theilliol, Dominique Sauter

\section{To cite this version:}

Mickael Rodrigues, Didier Theilliol, Dominique Sauter. Design of an active fault tolerant control for nonlinear systems described by a multi-model representation. The 20th IEEE International Symposium on Intelligent Control, ISIC'05 and the 13th Mediterranean Conference on Control and Automation, MED'05, Jun 2005, Limassol, Cyprus. pp.CDROM. hal-00364800

\section{HAL Id: hal-00364800 \\ https://hal.science/hal-00364800}

Submitted on 27 Feb 2009

HAL is a multi-disciplinary open access archive for the deposit and dissemination of scientific research documents, whether they are published or not. The documents may come from teaching and research institutions in France or abroad, or from public or private research centers.
L'archive ouverte pluridisciplinaire HAL, est destinée au dépôt et à la diffusion de documents scientifiques de niveau recherche, publiés ou non, émanant des établissements d'enseignement et de recherche français ou étrangers, des laboratoires publics ou privés. 


\title{
Design of an Active Fault Tolerant Control for Nonlinear Systems described by a Multi-Model Representation
}

\author{
Mickaël Rodrigues, Didier Theilliol and Dominique Sauter
}

\begin{abstract}
In this paper, an new active Fault Tolerant Control (FTC) strategy is developed to nonlinear systems described by multiple linear models to prevent the system deterioration by the synthesis of adapted controllers. When a fault is detected by the fault detection and diagnosis scheme, the reconfigurable controller is designed automatically using a robust gain scheduling strategy. The main contribution concerns the design of state feedback gains through $\mathcal{L} \mathcal{M}$ both in fault-free and faulty cases in order to preserve the system performances over a wide operating range. For each separate actuator with which the system is robustly stabilizable, a robust pole placement is designed by pole clustering. The effectiveness and performances of the method have been illustrated in simulation considering a classical nonlinear benchmark.
\end{abstract}

\section{INTRODUCTION}

The increasing demands for higher system performance, product quality, productivity and cost efficiency lead to a continuous growth of the complexity and automation degree of technical processes. Typical instances of such kind of complex and distributed systems are sophisticated vehicles, civil aircraft, environment treatment processes, large fleets and infrastructures [1]. Associated with these development trends, high reliability, availability and safety become an important system requirement which is included in many international standards and regulations. The objective of Fault Tolerant Control system (FTC) is to maintain current performances closed to desirable performances and preserve stability conditions in the presence of component and/or instrument faults; in some circumstances reduced performance could be accepted as a trade-off. Accommodation capability of a control system depends on many factors such as the severity of the failure, the robustness of the nominal system, and the actuators redundancy. FTC can be motivated by different goals depending on the application under consideration, for instance, safety in flight control or reliability or quality improvements in industrial processes. Various approaches for FTC have been suggested in the literature [2], [3], [4] and [5] but often deal with linear systems. For nonlinear systems, the design of Fault Tolerant controller is far more complicated. Nonlinear systems based on multiple linear models, represents an attractive solution to deal with the control of nonlinear systems [6], [7], [8] or FDI methods as in the chapter nine of [9] where nonlinear

This work was not supported by any organization

M. Rodrigues, D. Theilliol and D. Sauter are with Centre de Recherche en Automatique de Nancy - CNRS UMR 7039, Université Henri Poincaré, BP 239 - 54506 Vandoeuvre Cedex France. Phone: +33 $383684 \quad 465$ - Fax: +33 383684462 mickael.rodrigues@cran.uhp-nancy. fr dynamic systems are described by a number of locally linearized models based on the idea of Tagaki-Sugeno fuzzy models or as interpolated multiple linear models [10]. A great number of gain scheduling strategies are developed in fault-free case [6] and we proposed to develop one of them in faulty-case. Various recent FDI/FTC studies, based on a multiple model method have been developed in order to detect, isolate and estimated an accurate state of a system in presence of faults/failures around an unique operating point [11], [12] and in chapter 7 of [4]. Compared to multi-model based reconfigurable control method presented by [13], this paper not consider some redundant hardware which is very useful when failures are supposed to occur on the system. In this paper, an active fault tolerant strategy is developed so as to avoid actuator fault effect on nonlinear system where faults are assumed to be incipient, abrupt but not generate a total actuator fault i.e. a failure. Under the assumption that the fault is detected, isolated and estimated, the developed method preserves the system performances through an appropriate gain scheduling synthesis in faulty case. Compared to recent work applied to similar nonlinear system [14], where a multi-model representation is considered, the proposed FTC strategy is not based on an additional control law but on the redesign of appropriate gain in faulty case allowing stability and performances of the system.

The paper is organized as follows. In section II, we introduce a state space representation with both additive and multiplicative fault defined around operating points. A global state space representation of nonlinear system is given through a multi-model approach. In section III, we introduce a pole placement by $\mathcal{L} \mathcal{M I}$ region and then a gain synthesis for each actuator generate an active global state feedback synthesis. This scheme provides a new active Fault Tolerant Control synthesis. A simulation example is given in section IV to illustrate the proposed method. Finally, concluding remarks are given in the last section.

\section{NONLINEAR REPRESENTATION AND FAULT TOLERANT CONTROL STRATEGY}

\section{A. Nonlinear representation}

Consider a discrete-time nonlinear dynamical system described by:

$$
\left\{\begin{array}{cc}
x(k+1) & =g\left(x_{k}, u_{k}\right) \\
y(k) & =h\left(x_{k}, u_{k}\right)
\end{array}\right.
$$

where $x_{k} \in \mathcal{X} \in \mathbb{R}^{n}$ represents the state vector, $u_{k} \in$ $\mathcal{U} \in \mathbb{R}^{p}$ is the input vector and $y \in \mathbb{R}^{m}$ is the output 
vector. We assume that functions $g\left(x_{k}, u_{k}\right)$ and $h\left(x_{k}, u_{k}\right)$ are continuously differentiable.

Definition:[15] Given a set $\mathcal{U}$, a point $x_{0} \in \mathcal{X} \subseteq \mathbb{R}^{n}$ is an equilibrium point of the system (1) if a control $u_{0} \in$ $\mathcal{U}$ exists such that $x_{0}=g\left(x_{0}, u_{0}\right)$. We call a connected set of equilibrium points an equilibrium surface. Suppose $\left(x_{e}, u_{e}, y_{e}\right)$ is a point on an equilibrium surface and define a shifted state $\bar{x}=x-x_{e}$ and a shifted input $\bar{u}=u-u_{e}$, the nonlinear system with respect to $\left(x_{e}, u_{e}\right)$ can be expressed as:

$$
\left\{\begin{array}{c}
\left.\bar{x}(k+1)=g(x(k), u(k))-g\left(x_{e}, u_{e}\right)\right) \triangleq f(\bar{x}(k), \bar{u}(k)) \\
\left.\bar{y}(k)=h(x(k), u(k))-h\left(x_{e}, u_{e}\right)\right) \triangleq v(\bar{x}(k), \bar{u}(k))
\end{array}\right.
$$

It is assumed that dynamic behaviour of the system operating at different operating points can be approximated by a set of $N$ linear time invariant models in chapter 1 of [10] and in the following references [16], [8], [15]. Consider the following state space representation of a nonlinear system around $\mathrm{j}$-th operating point, $j \in[1, \ldots, N]$, with additive actuator faults:

$$
\begin{gathered}
x_{k+1}-x_{e}^{j}=A_{j}\left(x_{k}-x_{e}^{j}\right)+B_{j}\left(u_{k}-u_{e}^{j}\right)+F_{j} f_{k} \\
y_{k}-y_{e}^{j}=C_{j}\left(x_{k}-x_{e}^{j}\right)+D_{j}\left(u_{k}-u_{e}^{j}\right)
\end{gathered}
$$

Matrices $\left(A_{j}, B_{j}, C_{j}, D_{j}\right)$ are invariant matrices defined around the $j^{\text {th }}$ operating point $\left(\mathcal{O P}_{j}\right)$ generally obtained from a nonlinear system using a first-order Taylor expansion about $\left(x_{e}^{j}, u_{e}^{j}\right)$ or identification of a nonlinear system around predefined operating points as for example in chemical processes in [17], [18]. The fault distribution matrix is represented by $F_{j} \in \mathbb{R}^{n \times p} . f_{k} \in \mathbb{R}^{p}$ represents the actuator fault vector and in fault-free case it is obviously equal to zero. In the following, we consider that $D_{j}=0$. This linear system (3) can be specified by the set of system matrices as in fault-free case in [10], [8] and in faulty-case in [16]:

$$
S_{j}=\left[\begin{array}{lll}
A_{j} & B_{j} & F_{j} \\
C_{j} & &
\end{array}\right], \forall j=[1, \ldots, N]
$$

Let $S_{k}$ be a matrix sequence varying within a convex set, defined as:

$$
S_{k}:=\left\{\sum_{j=1}^{N} \rho_{k}^{j} S_{j}: \rho_{k}^{j} \geq 0, \sum_{j=1}^{N} \rho_{k}^{j}=1\right\}
$$

In the Multi-Model framework, $S_{k}$ characterizes at each sample the nonlinear system and consequently, the dynamic behavior of nonlinear system can be defined by a convex set of a multiple LTI models. As considered in [10] and [18], a possible model that would be able to catch the full range of operation is made from $N$ weighting local models $\mathcal{O} \mathcal{P}_{j}$ by interpolation functions $\rho_{k}^{j}$. These activation functions $\rho_{k}^{j} \forall j \in[1, \ldots, N]$ lie in a convex set $\Omega=$ $\left\{\rho_{k}^{j} \in \mathbb{R}^{N}, \rho_{k}=\left[\begin{array}{lll}\rho_{k}^{1} & \ldots \rho_{k}^{N}\end{array}\right]^{T}, \quad \rho_{k}^{j} \geq 0 \quad \forall j \quad\right.$ and $\left.\sum_{j=1}^{N} \rho_{k}^{j}=1\right\}$ and these functions are generated via works of [19] and [18], which permit to generate insensitive residual to faults and some uncertainties. So, activation functions are robust against faults and errors modeling and the dynamic system is well represented. The representation
(3) considers additive fault representation but there exists multiplicative representation for specific actuator fault as:

$$
\begin{aligned}
x_{k+1} & =A_{j}\left(x_{k}-x_{e}^{j}\right)+B_{j}\left(I-\gamma^{a}\right)\left(u_{k}-u_{e}^{j}\right)+x_{e}^{j} \\
y_{k} & =C_{j}\left(x_{k}-x_{e}^{j}\right)+y_{e}^{j}
\end{aligned}
$$

So, let consider a local multiplicative actuator fault representation as a blended representation (as in [8] in fault-free

$$
\begin{aligned}
x_{k+1} & =\sum_{j=1}^{N} \rho_{k}^{j}\left[A_{j}\left(x_{k}-x_{e}^{j}\right)+B_{j}\left(I-\gamma^{a}\right)\left(u_{k}-u_{e}^{j}\right)+x_{e}^{j}\right] \\
y_{k} & =\sum_{j=1}^{N} \rho_{k}^{j}\left[C_{j}\left(x_{k}-x_{e}^{j}\right)+y_{e}^{j}\right]
\end{aligned}
$$

with $\gamma^{a} \triangleq \operatorname{diag}\left[\gamma_{1}^{a}, \gamma_{2}^{a}, \ldots, \gamma_{p}^{a}\right], \gamma_{i}^{a} \in \mathbb{R}$, such that $\gamma_{i}^{a}=1$ represents a total lost, a failure of i-th actuator, $i \in[1, \ldots, p]$ and $\gamma_{i}^{a}=0$ implies that i-th actuator operates normally. The objective of fault detection and diagnosis is to determine the extent of the loss in the control effectiveness by estimating $\gamma_{i}^{a}$ on-line in real-time [16] so that an on-line automatic reconfigurable controller can be synthesized. The matrices $\left(A_{j}, B_{j}, C_{j}\right) \in S_{k}$ and the relation between state space representations (3) and (6) is equivalent to

$$
F_{j} f_{k}=-B_{j} \gamma^{a}\left(u_{k}-u_{e}^{j}\right)
$$

\section{B. Fault Tolerant Control design}

In closed-loop, the fault occurrence could be detected as described in [1] for linear case and [14] for multi-linear systems and Fault Tolerant Control could be performed via an additional control law as in [20] which permits to avoid fault on a system based on a state space representation as (3). In these papers, the goal was to synthesize a new control law $u_{F T C}$ with a nominal one $u_{\text {nom }}$ and additional one $u_{a d}$. The term $u_{a d}$ was performed in order to vanish fault on the system. The global control law is obtained by interpolating gains of each local controller [6] and is defined as:

$$
u_{F T C}^{j}=u_{n o m}^{j}+u_{a d}^{j}
$$

with $u_{n o m}^{j}=-K_{n o m}\left(x_{k}-x_{e}^{j}\right)+u_{e}^{j}$. The gains were only performed for nominal cases and do not take into account fault occurrence. Under the assumption that fault is detected, isolated and estimated, based on a multiplicative fault representation defined in (7), we propose a control law $u_{F T C}^{j}$, around an Operating Point $j$, which must vanish faults as the following form:

$$
u_{F T C}^{j}=-\left(I-\gamma^{a}\right)^{+} K\left(x_{k}-x_{e}^{j}\right)+u_{e}^{j}
$$

The objective of fault accommodation consists in a strategy by which the faulty system is controlled in a specific way, so as to achieve the objectives which were (before the fault) achieved by the healthy system. So, note that

$$
\begin{aligned}
& u_{F T C}^{j}(k)=\left[I-\gamma^{a}\right]^{+} u_{n o m}^{j}(k)=-\left[I-\gamma^{a}\right]^{+} K_{n o m}\left(x_{k}-\right. \\
& \left.x_{e}^{j}\right)+u_{e}^{j}=-K_{F T C}\left(x_{k}-x_{e}^{j}\right)+u_{e}^{j}
\end{aligned}
$$

In the following, we will note $u_{F T C}^{j}(k)$ as $u_{F T C}^{j}$ so as to relax notations and have a better understanding. We note $(.)^{+}$the Moore-Penrose matrix inverse and it is assumed 
that matrix $\left[I-\gamma^{a}\right]$, which is diagonal, has full column rank i.e. the dimension $n$ of the system is greater than number of $p$ actuators. Assuming that there is no mismatch between fault and their estimation and without considering total fault, this state feedback is inserted in the state space representation (6) around an $\mathcal{O P}_{j}$ and leads to:

$$
\begin{aligned}
x_{k+1}-x_{e}^{j} & =A_{j}\left(x_{k}-x_{e}^{j}\right)+B_{j}\left(I-\gamma^{a}\right)\left(-K_{F T C}\left(x_{k}-x_{e}^{j}\right)\right. \\
& \left.+u_{e}^{j}-u_{e}^{j}\right) \\
& =\left(A_{j}-B_{j} K_{n o m}\right)\left(x_{k}-x_{e}^{j}\right)
\end{aligned}
$$

Even if there is an actuator fault on the system, this one still operates normally with the new state feedback gain $K_{F T C}=\left[I-\gamma^{a}\right]^{+} K_{\text {nom }}$. As previously defined in (5), the model probability is viewed as a scheduled variable in the synthesis of the controller like in [6], [8] and [14], and the new global control law is defined as:

$$
u_{k}=\sum_{j=1}^{N} \rho_{k}^{j} u_{F T C}^{j}=-\sum_{j=1}^{N} \rho_{k}^{j}\left[K_{F T C}\left(x_{k}-x_{e}^{j}\right)+u_{e}^{j}\right]
$$

with $u_{F T C}^{j}$ the output of each local controller defined around each $\mathcal{O P}_{j}$. In order to synthesize state feedback for FTC ensuring both active control in multi-model philosophy and quadratic stability, use of $\mathcal{L M I}$ provide a well toolbox for these purposes. Total failures are not consider in this paper but only partial ones. We attract attention that the system has to be observable in all around each operating points.

\section{Fault Tolerant Control on multiple OPERATING POINTS}

\section{A. Pole clustering}

In the synthesis of control system, some desired performances should be considered in addition to stability. In fact, classical stability conditions do not deal with transient responses of the closed-loop system. In contrast, a satisfactory transient response can be guaranteed by confining its poles in a prescribed region. For many real problems, exact pole assignment may not be necessary: it suffices to locate the closed-loop poles in a prescribed subregion in the complex plane. We will discuss about pole clustering by introducing the following $\mathcal{L M I}$-based representation of stability regions.

Definition 1: LMI stability region [21]. A subset D of the complex plane is called an LMI region if there exist a symmetric matrix $\alpha=\left[\alpha_{k l}\right] \in \mathbb{R}^{n \times n}$ and a matrix $\beta=$ $\left[\beta_{k l}\right] \in \mathbb{R}^{n \times n}$ such that

$$
\mathcal{D}=\left\{z \in C: f_{\mathcal{D}}(z)<0\right\}
$$

where the characteristic function $f_{\mathcal{D}}(z)$ is given by

$$
f_{\mathcal{D}}(z)=\left[\alpha_{k l}+\beta_{k l} z+\beta_{l k} \bar{z}\right]_{1 \leq k, l \leq n}
$$

( $f_{\mathcal{D}}$ is valued in the space of $n \times n$ Hermitian matrices). $\square$ Moreover, $\mathcal{L M I}$ regions are convex and symmetric with

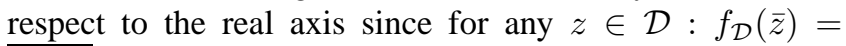
$\overline{f_{\mathcal{D}}(z)}<0$. Then, pole location in a given $\mathcal{L} \mathcal{M I}$ region can be characterized in terms of a block matrix if and only if there exists a symmetric matrix $P>0$ such that [21]:

$$
\begin{aligned}
& M_{\mathcal{D}}\left(A_{c l}, P\right)=\alpha \otimes P+\beta \otimes\left(A_{c l} P\right)+\beta^{T} \otimes\left(A_{c l} P\right)^{T} \\
& =\left[\alpha_{k l} P+\beta_{k l} A_{c l} P+\beta_{l k} P A_{c l}^{T}\right]_{1 \leq k, l \leq m}
\end{aligned}
$$

with $A_{c l}=A-B K$ and $M=\left[\mu_{k l}\right]_{1 \leq k, l \leq n}$ means that $M$ is an $n \times n$ matrix (respectively, bloc matrix) with generic entry (respectively bloc) $\mu_{k l}$. Note that $M_{\mathcal{D}}\left(A_{c l}, P\right)$ in (16) and $f_{\mathcal{D}}(z)$ in (15) are related by the substitution $\left(P, A_{c l} P, P A_{c l}^{T}\right) \leftrightarrow(1, z, \bar{z})$. It is easily seen that $\mathcal{L} \mathcal{M I}$ regions are convex and symmetric with respect to real axis. Specifically, the circular $\mathcal{L} \mathcal{M I}$ region $\mathcal{D}$ is considered:

$$
\mathcal{D}=\left\{x+j y \in C:(x+q)^{2}+y^{2}<r^{2}\right\}
$$

centered at $(-q, 0)$ with radius $r>0$, where the characteristic function $f_{\mathcal{D}}(z)$ is given by:

$$
f_{D}(z)=\left(\begin{array}{cc}
-r & \bar{z}+q \\
z+q & -r
\end{array}\right)
$$

Therefore, this circular region puts a lower bound on both the exponential decay rate and the damping ratio of the closed-loop response, and thus is very common in practical control design. It is obvious that well chosen $\mathcal{L M} \mathcal{I}$ region is needed for ensuring stability and good results: the parameters $q, r$ have to be defined by the engineer.

\section{B. Control law synthesis in fault-free case}

Let consider the state space representation (7) of nonlinear system defined around the Operating Points $\mathcal{O P}_{j}$, $\forall j=1, \ldots, N$

$$
\begin{aligned}
x_{k+1} & =\sum_{j=1}^{N} \rho_{k}^{j}\left[A_{j}\left(x_{k}-x_{e}^{j}\right)+\sum_{i \in \mathcal{I}} B_{j}^{i}\left(I-\gamma^{a}\right)\left(u_{k}-u_{e}^{j}\right)+x_{e}^{j}\right] \\
y_{k} & =\sum_{j=1}^{N} \rho_{k}^{j}\left[C_{j}\left(x_{k}-x_{e}^{j}\right)+y_{e}^{j}\right]
\end{aligned}
$$

with $\mathcal{I}: i=1, \ldots, p$ the actuators for each $\mathcal{O P}_{j}$ and matrices $\left(A_{j}, B_{j}, C_{j}\right) \in S_{k}$ defined in (5). Consider the matrix representing total faults in all actuators but the $\mathrm{i}$-th:

$$
B_{j}^{i}=\left[0, \ldots, 0, b_{j}^{i}, 0, \ldots, 0\right]
$$

and $B_{j}=\left[b_{j}^{1}, b_{j}^{2}, \ldots, b_{j}^{p}\right.$, with $b_{j}^{i} \in \mathbb{R}^{n \times 1}$. It is assumed that each column of $B_{j}$ is full column rank whatever the $\mathcal{O P} \mathcal{P}_{j}$. The pairs $\left(A_{j}, b_{j}^{i}\right), \forall i=1, \ldots, p$ are assumed to be controllable for all $\forall j=1, \ldots, N$. Let $\mathcal{D}$, a $\mathcal{L} \mathcal{M I}$ region defining a disk with a center $(-q, 0)$, and a radius $r$ with $(q+r)<1$ for defining pole assignment in the unit circle. Assume that for each $B_{j}^{i}$, there exist matrices $X_{i}=X_{i}^{T}>0$ and $Y_{i}, \forall j=1, \ldots, N, \forall i=1, \ldots, p$ such as:

$$
\left(\begin{array}{cc}
-r X_{i} & q X_{i}+\left(A_{j} X_{i}-B_{j}^{i} Y_{i}\right)^{T} \\
q X_{i}+A_{j} X_{i}-B_{j}^{i} Y_{i} & -r X_{i}
\end{array}\right)<0
$$

It can be noticed that if $q=0$ and $r=1$, the previous equation (21) is equivalent to solve a classical quadratic stability problem. Based on the assumptions that for each $\mathcal{O P}{ }_{j}$ each pairs $\left(A_{j}, b_{j}^{i}\right)$ are controllable, it is possible to find a Lyapunov matrices $X_{i}>0$ and state-feedback $K_{i}$ with $Y_{i}=K_{i} X_{i}$ and finally form a global state-feedback gain $K_{\text {nom }}$. 
Theorem 1: Consider the system (19) in fault-free case $\left(\gamma^{a}=0\right)$ defined for all $\mathcal{O} \mathcal{P}_{j}, j=1, \ldots, N$ : it is possible to develop a mixing of pre-designed state-feedback gains matrices $K_{i}=Y_{i} X_{i}^{-1}$ for each actuator $i$ with $i=1, \ldots, p$ such that (21) holds for all $j=1, \ldots, N$. The state feedback control for each operatipg point is given by:

$$
\begin{aligned}
u_{n o m}^{j} & =-\left(\sum_{i=1} G_{i} Y_{i}\right)\left(\sum_{i=1} X_{i}\right)^{-1}\left(x_{k}-x_{e}^{j}\right)+u_{e}^{j} \\
& =-Y X^{-1}\left(x_{k}-x_{e}^{j}\right)+u_{e}^{j}=-K_{n o m}\left(x_{k}-x_{e}^{j}\right)+u_{e}^{j}
\end{aligned}
$$

with $\sum_{i=1}^{p} G_{i} Y_{i}=Y, X=\sum_{i=1}^{p} X_{i}$ and $G_{i}=B_{j}^{i+} B_{j}^{i}$ is matrix that has zeros everywhere except in entry $(i, i)$ where it has a one. The general control law for all $\mathcal{O P}_{j}$ could be defined as:

$$
u_{k}=\sum_{j=1}^{N} \rho_{k}^{j} u_{n o m}^{j}=-\sum_{j=1}^{N} \rho_{k}^{j}\left[K_{n o m}\left(x_{k}-x_{e}^{j}\right)+u_{e}^{j}\right]
$$

Proof:

Summation of (21) for $i=1, \ldots, p$ gives for one equilibrium point $j$

$\sum_{i=1}^{p}\left(\begin{array}{cc}-r X_{i} & q X_{i}+\left(A_{j} X_{i}-B_{j}^{i} Y_{i}\right)^{T} \\ q X_{i}+\left(A_{j} X_{i}-B_{j}^{i} Y_{i}\right) & -r X_{i}\end{array}\right)<0$

related to the quadratic $\mathcal{D}$-stability in a prescribed $\mathcal{L} \mathcal{M I}$ region. Next, denote $X=\sum_{i=1}^{p} X_{i}$ (with $X=X^{T}>0$ ) to obtain

$$
\left(\begin{array}{cc}
-r X & q X+\left(A_{j} X-\sum_{i=1}^{p} B_{j}^{i} Y_{i}\right)^{T} \\
q X+\left(A_{j} X-\sum_{i=1}^{p} B_{j}^{i} Y_{i}\right) & -r X
\end{array}\right)<0
$$

Now, denote the 1-th row of the matrix $Y_{i}$ as $Y_{i}^{l}, i=1, \ldots, p$ and $l=1, \ldots, p$, i.e. $\quad Y_{i}^{l}=G_{l} Y_{i}$

Therefore,

$$
\sum_{i=1}^{p} B_{j}^{i} Y_{i}=\sum_{i=1}^{p}\left[0, \ldots, 0, b_{j}^{i}, 0, \ldots, 0\right] Y_{i}^{i}=B_{j} \sum_{i=1}^{p} Y_{i}^{i}
$$

leading to

$$
\sum_{i=1}^{p} B_{j}^{i} Y_{i}=B_{j}\left(\sum_{i=1}^{p} G_{i} Y_{i}\right)
$$

Thus, taking $Y=\sum_{i=1}^{p} G_{i} Y_{i}$, equation (28) becomes

$$
\sum_{i=1}^{p} B_{j}^{i} Y_{i}=\sum_{i \in \mathcal{I}} B_{j}^{i} Y_{i}=B_{j} Y
$$

which, substituted into $\mathcal{L} \mathcal{M I}$ (25), finally makes

$$
\left(\begin{array}{c}
-r X q X+\left(A_{j} X-B_{j} Y\right)^{T} \\
q X+\left(A_{j} X-B_{j} Y\right)-r X
\end{array}\right)<0
$$

for all $\mathcal{O P}_{j}, j=1, \ldots, N$. By multiplying each $\mathcal{L} \mathcal{M I}$ (30) by $\rho_{k}^{j}$ and summing all of them, we obtain

$$
\left(\begin{array}{cc}
-r X & q X+\sum_{j=1}^{N} \rho_{k}^{j}\left(A_{j} X-B_{j} Y\right)^{T} \\
q X+\sum_{j=1}^{N} \rho_{k}^{j}\left(A_{j} X-B_{j} Y\right) & -r X
\end{array}\right)<0
$$

it is equivalent to

$$
\left(\begin{array}{cc}
-r X & q X+(A(\rho) X-B(\rho) Y)^{T} \\
q X+(A(\rho) X-B(\rho) Y) & -r X
\end{array}\right)<0
$$

with $A(\rho)=\sum_{j=1}^{N} \rho_{k}^{j} A_{j} \in S_{k}$ and $B(\rho)=\sum_{j=1}^{N} \rho_{k}^{j} B_{j} \in$ $S_{k}$. Hence quadratic $\mathcal{D}$-stability is ensured by solving (31) and $Y=K_{n o m} X$ quadratically stabilizes the system (19) under the set $S_{k}$ by solving (32) with a state feedback law $u_{k}=\sum_{j=1}^{N} \rho_{k}^{j} u_{\text {nom }}^{j}=-\sum_{j=1}^{N} \rho_{k}^{j}\left[Y X^{-1}\left(x_{k}-x_{e}^{j}\right)+u_{e}^{j}\right]$.

Remark1: It could be noticed that gain synthesis through multiple operating point with such $\mathcal{L} \mathcal{M I}$ consideration provide only one single gain for all $\mathcal{O P}$ due to Bilinear Matrix inequality $(\mathcal{B M I})$ problem in term $(2,1)$ of $\mathcal{L} \mathcal{M I}$ (21). However, other system such piecewise linear system could use the same approach with a multiple gain synthesis as in [17].

Remark2: If all local models have the same $B_{j}$ matrices, i.e. $B_{j}=B$ for all $j=1, \ldots, N$, it is also possible to use the following parameter-dependent state feedback law instead of (23):

$$
u_{k}=\sum_{j=1}^{N} \rho_{k}^{j} u_{n o m}^{j}=-\sum_{j=1}^{N} \rho_{k}^{j}\left[K_{j}\left(x_{k}-x_{e}^{j}\right)+u_{e}^{j}\right]
$$

with $K_{j}=Y_{j} Q^{-1}$. The resulting matrices remain the same except that $K_{j}$ replaces $K, B$ replaces $B_{j}$ and $Y_{j}$ replaces $Y$.

\section{Active Fault Tolerant Control design}

By considering the system (19) and based on the previous synthesis control law, the FTC method can be developed in this section where only partial actuator faults are considered under assumptions that fault occurrence and fault magnitude $\gamma^{a}$ are exactly known.

Theorem 2: Consider the system (19) in faulty case $\left(\gamma^{a} \neq 0\right)$ coupled with regulators with gains $K_{i}=Y_{i} X_{i}^{-1}$ for all equilibrium point $j=1, \ldots, N$ and for each actuator $i$ with $i=1,2, . ., p$. Let introduce the set of indexes of all actuators that are not completely lost, i.e.

$$
\Theta \triangleq\left\{i: i \in(1,2, . ., p), \gamma_{i}^{a} \neq 1\right\}
$$

The control action is

$$
u_{F T C}^{j}=-\left(I-\gamma^{a}\right)^{+}\left(\sum_{i \in \Theta} G_{i} Y_{i}\left(\sum_{i \in \Theta} X_{i}\right)^{-1}\right)\left(x_{k}-x_{e}^{j}\right)+u_{e}^{j}
$$

where $G_{i}=B_{j}^{i+} B_{j}^{i}$, applied to the faulty system allows to constrain pole placement in prescribed $\mathcal{L} \mathcal{M I}$ region.

Proof: Applying the new control law (34) to the faulty system (7), leads to the following equation

$$
B_{j}\left(I-\gamma^{a}\right) u_{F T C}^{j}=B_{j} \Gamma^{a}\left(\sum_{i \in \Theta} G_{i} Y_{i}\right)\left(\sum_{i \in \Theta} X_{i}\right)^{-1}\left(x_{k}-x_{e}^{j}\right)+u_{e}^{j}
$$

with

$$
\Gamma^{a}=\left(\begin{array}{cc}
I_{p-h} & 0 \\
0 & O_{h}
\end{array}\right)
$$

$\Gamma^{a}$ is a diagonal matrix which contains only entries zero (representing total faults) and one (no fault). But here $h=0$, which is the number of actuators completely lost, 
due to the fact that only the set $\Theta$ is considered. Since $B_{j} \Gamma^{a}=\sum_{i \in \Theta} B_{j}^{i}$ models only the actuators that are not completely lost, then performing the summations in the proof of Theorem (1) over the elements of $\Theta$ shows that $\left(\sum_{i=1}^{p} G_{i} Y_{i}\right)\left(\sum_{i=1}^{p} X_{i}\right)^{-1}$ is the state-feedback gain matrix for the faulty system $\left(A_{j}, \sum_{i \in \Theta} B_{j}^{i}, C_{j}\right)$.

The control law in equation (34) implies that

$$
u_{F T C}^{j}=-K_{F T C}\left(x_{k}-x_{e}^{j}\right)+u_{e}^{j}
$$

with

$$
K_{F T C}=\left(I-\gamma^{a}\right)^{+} \sum_{i \in \Theta} G_{i} Y_{i}\left(\sum_{i \in \Theta} X_{i}\right)^{-1}
$$

The global control law $U_{F T C}$ of the system is realized as:

$$
\begin{aligned}
u_{k} & =\sum_{j=1}^{N} \rho_{k}^{j} u_{F T C}^{j} \\
& =-\sum_{j=1}^{N} \rho_{k}^{j}\left[K_{F T C}\left(x_{k}-x_{e}^{j}\right)+u_{e}^{j}\right]
\end{aligned}
$$

\section{APPLICATION}

\section{A. Process description}

The approach presented in this paper has been applied to the well known three tanks benchmark as in [14]. As all the three liquid levels are measured by level sensors, the output vector $Y$ is $\left[\begin{array}{lll}l_{1} & l_{2} & l_{3}\end{array}\right]^{T}$. The control input vector is $U=\left[\begin{array}{ll}q_{1} & q_{2}\end{array}\right]^{T}$. The goal is to control the system around three equilibrium points. Thus, 3 linear models have been identified around each of these equilibrium points and the operating conditions are given in Table (I). The linearized

TABLE I

\begin{tabular}{|c|c|c|c|}
\hline Operating point & $\mathrm{n} 1$ & $\mathrm{n} 2$ & $\mathrm{n} 3$ \\
\hline & $0.20 ;$ & $0.50 ;$ & $0.50 ;$ \\
$y_{e}^{j}(m)$ & $0.15 ;$ & $0.15 ;$ & $0.405 ;$ \\
& 0.175 & 0.325 & 0.45 \\
\hline$u_{e}^{j}$ & $1.7509 ;$ & $4.6324 ;$ & $2.4761 ;$ \\
$\left(m^{3} / s\right) \times 10^{-5}$ & 4.0390 & 1.1574 & 6.9787 \\
\hline
\end{tabular}

system is described by a discrete state space representation with a sampling period $T s=1 s$. For each $\mathcal{O P}$, each control matrix pair $\left(A_{j}, b_{j}^{i}\right)$ is controllable. Controllers have been designed for levels $l_{1}$ and $l_{2}$ to track reference input vector $Y^{r} \in \mathbb{R}^{2}$. Nominal controllers have been designed through Theorem (1), leading to two state feedback gain matrices $K_{1}, K_{2}$ (due to 2 actuators) for all the three $\mathcal{O P}$ in order to achieve satisfying tracking performances. The simulation of actuator faults on the system does not affect the controllability and observability of the system.

\section{B. Results and comments}

Simulations have been performed such as the 3 operating conditions described in Table (I) are reached and weighting functions for each local model are presented in figure (1) always close to the dynamic behaviour of the nonlinear system according to the considered operating regimes. Figure (2) shows the time history of the outputs with respect to set-point changes occur at time instant $500 \mathrm{~s}$ and after at time instant $2500 \mathrm{~s}$. In the simulation, gaussian

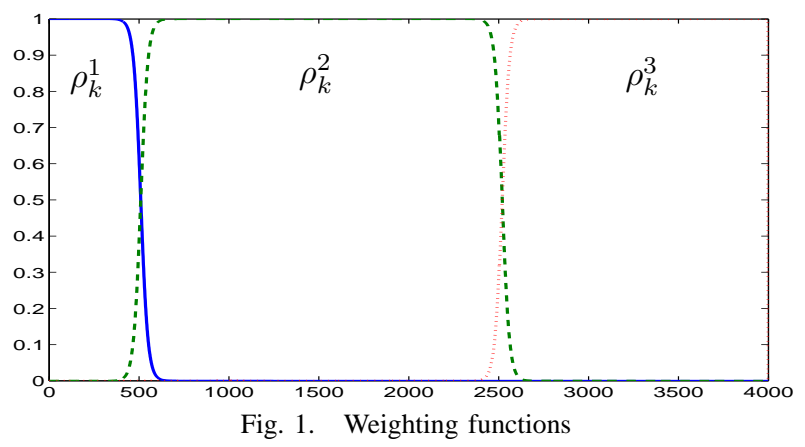

noises $\left(N\left(0,1 e^{-4^{2}}\right)\right)$ are added to each output signal. The reference inputs correspond to step changes for $l_{1}$ and $l_{2}$. The consequence of an actuator fault is illustrated in

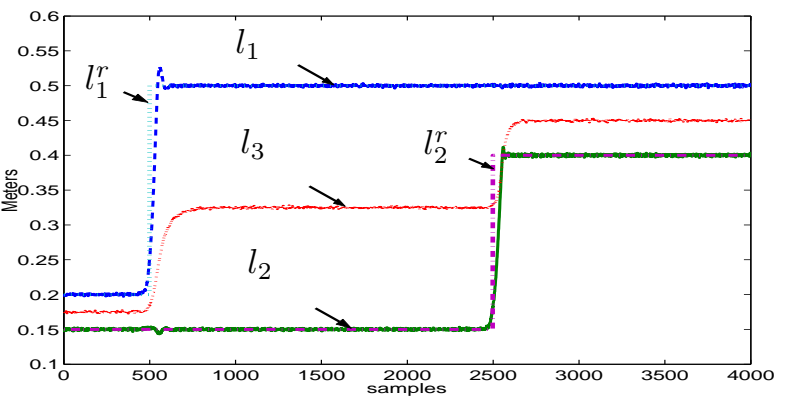

Fig. 2. System outputs in fault-free case with a nominal control law

figure (3). A gain degradation of pump 1 (clogged or rusty pump) equivalent to $80 \%$ loss of effectiveness is supposed to occur at time instant 1000 s. Consequently, the dynamic behaviour of the other levels is also affected by this fault and control system tries to cancel the static error created by the corrupted input. Consequently, the real output is different from the reference input and the control law is different from its nominal value. Since an actuator fault acts on the system as a perturbation, and in spite of the presence of an integral controller, the system outputs can not reach again their nominal values. In the same way, the actuator Fault

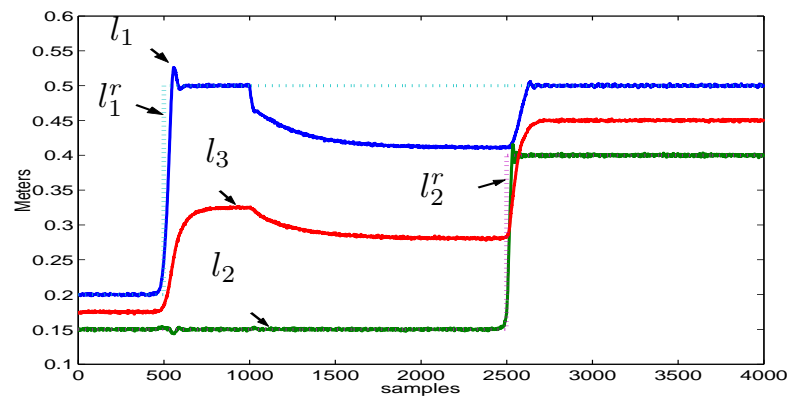

Fig. 3. System outputs with pump degradation and a nominal controller

Tolerant Control method's ability to compensate faults is illustrated in the presence of the same fault. Once the fault is isolated and simultaneously estimated, a new control law (39) is computed in order to reduce the fault effect on the system. Indeed, since the effect of an actuator fault 
is quite similar to the effect of a perturbation, the system outputs reach again their nominal values, as illustrated in figure (4). A time delay between fault occurrence and fault compensation equals to 10 samples is considered in our simulation. Computation of the tracking error norm

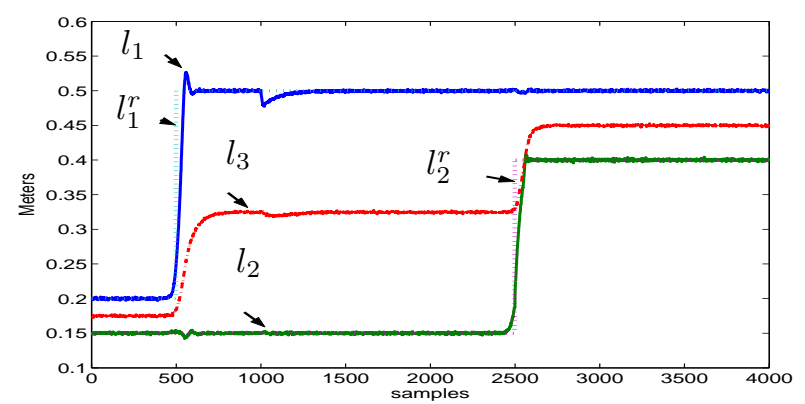

Fig. 4. System outputs with pump degradation and a FTC controller

in fault-free case, in faulty case without and with FTC underlines the performances of this approach as seen in Table (II). With FTC, the tracking error norm for output $l_{1}$ is a bit larger than with the fault-free case but it still widely smaller than the one without FTC. The actuator Fault Tolerant Control is able to maintain performances as close as possible to nominal ones and to ensure the closed-loop stability despite the presence of instruments malfunction. Remark: This proposed scheme could have an interesting

TABLE II

\begin{tabular}{|c|c|c|c|}
\hline \multirow{2}{*}{$\begin{array}{c}\text { Error } \\
\text { norm }\end{array}$} & Fault-free case & \multicolumn{2}{|c|}{ Actuator fault } \\
& & without FTC & with FTC \\
\hline$e_{l 1}$ & 1.1063 & 3.3365 & 1.1176 \\
\hline
\end{tabular}

development in aeronautic, for example, where redundant actuators are available and where a wide operating range is considered.

\section{CONClusion}

The method developed in this paper emphasises the importance of the active Fault Tolerant Control of nonlinear systems based on multi-model representation. This method is suitable for partial actuator faults on a wide operating range of the system. A robust controller is designed for each separate actuator through an $\mathcal{L} \mathcal{M I}$ pole placement in faultfree case and faulty case. It allows the system to continue operating safely, to avoid stopping it immediately and to ensure stability. The synthesis of this active state feedback control takes into account the information provided on-line by FDI scheme. The performances and the effectiveness of this active Fault Tolerant Control based on a multiple model approach have been illustrated through a nonlinear benchmark. Futures works will deal with total failures, restructuration and will improve the limits of the strategy.

\section{REFERENCES}

[1] H. Noura, D. Sauter, F. Hamelin, and D. Theilliol, "Fault-tolerant control in dynamic systems: Application to a winding machine," IEEE Control Systems Magazine, pp. 33-49, 2000.
[2] R. Patton, "Fault-tolerant control: the 1997 situation," In proc. IFAC Symposium Safeprocess, Kingston Upon Hull, U.K, vol. 2, pp. 10331055,1997

[3] Y. Zhang and J. Jiang, "Bibliographical review on reconfigurable Fault-Tolerant Control systems," In proc. IFAC Symposium Safeprocess, Washington. D.C, USA, CD-Rom, 2003.

[4] M. Blanke, M. Kinnaert, J. Lunze, and M. Staroswiecki, Diagnosis and Fault-Tolerant Control. Edts Springer-Verlag, 2003.

[5] Y. Zhang and J. Jiang, "Fault Tolerant Control system design with explicit consideration of performance degradation," IEEE Transactions on Aerospace and Electronic Systems, vol. 39, no. 3, pp. 838-848, July 2003.

[6] D. J. Leith and W. E. Leithead, "Survey of gain-scheduling analysis and design," International Journal of Control, vol. 73, no. 11, pp. 1001-1025, 2000.

[7] A. Banerjee, Y. Arkun, R. Pearson, and B. Ogunnaike, " $H_{\infty}$ control of nonlinear processes using multiple linear models," In Proc. ECC, Roma, Italy, pp. 2671-2676, 1995.

[8] A. Tayebi and M. Zaremba, "Iterative learning control for nonlinear systems described by a blended multiple model representation," International Journal of Control, vol. 75, no. 16/17, pp. 1376-1384, 2002.

[9] J. Chen and R. Patton, Robust model-based fault diagnosis for dynamic systems. Kluwer Academic Publishers, 1999.

[10] R. Murray-Smith and T. Johansen, Multiple Model Approaches to Modelling and Control. Taylor and Francis, 1997.

[11] P. Maybeck, "Multiple model adaptive algorithms for detecting and compensating sensor and actuator/surface failures in aircraft flight control systems," International Journal of Robust and Nonlinear Control, vol. 9, pp. 1050-1070, 1999.

[12] Y. Zhang and J. Jiang, "Integrated active Fault-Tolerant Control using IMM approach," IEEE Transactions on Aerospace and Electronics Systems, vol. 37, no. 4, pp. 1221-1235, 2001.

[13] K. Astrom, P. Albertos, M. Blanke, A. Isidori, and W. Schaufelberger, Control of complex systems. Edts Springer Verlag, 2001.

[14] D. Theilliol, D. Sauter, and J. Ponsart, "A multiple model based approach for Fault Tolerant Control in nonlinear systems," In proc. IFAC Symposium Safeprocess, Washington .D.C, USA, CD-Rom, 2003.

[15] Z. Wan and M. Kothare, "Efficient scheduled stabilizing model predictive control for constrained nonlinear systems," International Journal of Robust and Nonlinear Control, vol. 13, pp. 331-346, 2003.

[16] M. Rodrigues, M. Adam-Medina, D. Theilliol, and D. Sauter, "Fault Diagnosis on Industrial Systems based on a Multiple Model approach," In proc. IFAC Symposium on Automation in Mining, Mineral and Metal processing, Nancy, France, 2004.

[17] L. Ozkan, M. Kothare, and C. Georgakis, "Control of a solution copolymerization reactor using multi-model predictive control," Chemical Engineering Science, pp. 1207-1221, 2003.

[18] D. Theilliol, M. Rodrigues, M. Adam-Medina, and D. Sauter, "Adaptive filter design for FDI in nonlinear systems based on multiple model approach," In proc. IFAC Symposium Safeprocess, Washington D.C, USA, CD Rom, 2003.

[19] M. Adam-Medina, M. Rodrigues, D. Theilliol, and H. Jamouli, "Fault diagnosis in nonlinear systems through an adaptive filter under a convex set representation," In proc. ECC, Cambridge, U.K, CD Rom, 2003.

[20] D. Theilliol, H. Noura, and J. Ponsart, "Fault diagnosis and accommodation of three-tank system bsaed on analytical redundancy," ISA Transactions, vol. 41, pp. 365-382, 2002.

[21] M. Chilali and P. Gahinet, " $H_{\infty}$ design with pole placement constraints: an LMI approach," IEEE Trans. on Automatic Control, vol. 41, no. 3, pp. 358-367, 1996. 